\title{
TRADUIRE DES CONTES POUR LES ENFANTS - REFLEXIONS ET PRATIQUES ${ }^{1}$
}

\author{
Muguraș Constantinescu* \\ L’Université Stefan cel Mare/ Roumanie
}

\begin{abstract}
Résume: Dans le présent article nous prenons pour objet la spécificité de la traduction de jeunesse, en mettant l'accent sur la dimension culturelle à préserver dans le texte cible. Après un bref regard historique et théorique sur la traduction du texte pour enfants, nous présentons quelques analyses sommaires sur un corpus de contes pour enfants qui privilégient le rapport entre identité et altérité, l'ouverture à l'autre. Nous nous arrêtons, à travers notre pratique traductive, aux stratégies et procédés appropriés pour rendre la dimension culturelle du texte de départ afin de donner accès au jeune lecteur à une culture étrangère. Les stratégies traductives sont analysées en complémentarité avec des stratégies éditoriales et pédagogiques. Une place particulière est accordée à l'appareil paratextuel qui propose diverses formules pour satisfaire la curiosité du lecteur et contribue au développement de sa compétence encyclopédique.
\end{abstract}

Mots clefs: Spécificité. Dimension culturelle. Altérité. Paratexte. Compétence encyclopédique.

\footnotetext{
* Diplômée en langue et littérature françaises à l'Université A. I. Cuza, Iasi, docteur ès lettres de l'Université de Bucarest. Est professeur de littérature française et de la traduction littéraire à l'Université "SStefan cel Mare » de Suceava. Elle est rédactrice en chef de la revue Atelier de Traduction, directrice du Centre de Recherches INTER LITTERAS, coordinatrice du master Théorie et Pratique de la Traduction. Elle est coordinatrice de plusieurs projets de recherche exploratoire: CNCS PN-II-ID-PCE-2011-3-0812 (Projet de recherche exploratoire) Traduction culturelle et littérature(s) francophones : histoire, réception et critique des traductions. En 1997 elle a été lauréate de l'Institut International Charles Perrault d'Eaubonne pour le meilleur article inédit. Suceava, Roumanie.

E-mail:mugurasc@gmail.com
} 


\title{
TRANSLATING CHILDREN'S STORIES - REFLECTIONS AND PRACTICES
}

\begin{abstract}
The present article is concerned with the specificity of translations for children with an emphasis on the cultural dimension to be preserved in the target text. Following a brief historical and theoretical overview on the issue of translating texts for children, we undertake a succint analysis on a corpus made up of tales which display an overtness to the other while treating the identity-alterity issue. Starting from our own translation practice, we will insist upon those strategies and techniques fit to render the cultural dimension of the source text in order to give young readers access to a foreign culture. Translational strategies are analysed along editorial and pedagogical strategies, with a special focus on the paratext which aims at satisfying the readers' curiosity and contributing to the development of their encyclopedic competence.
\end{abstract}

Keywords: Specificity. Cultural dimension. Alterity. Paratext. Encyclopedic competence.

\section{Introduction}

Dans un entretien accordé à la revue Atelier de traduction, Tahar Ben Jelloun parle de la difficulté et de la responsabilité d'écrire pour les enfants : «Écrire pour les enfants est un travail à part. Il s'agit d'être pédagogue tout en étant créateur. Il faut être rigoureux, très exigeant car les enfants sont attentifs à la création. Il faut respecter leur attente. Il faut être clair, précis, juste. Il faut vérifier ce qu'on fait. » $(2007,17)$.

A travers notre expérience de traductrice et d'enseignante des théories de la traduction littéraire, nous avons perçu les paroles de Ben Jelloun sur l'écriture pour les enfants comme très proches d'un processus comparable, notamment, celui de traduire pour les enfants qui suppose un perpétuel exercice d'équilibre entre sous-traduction et sur-traduction, entre sous-estimation et sur-estimation des compétences du jeune lecteur. 


\section{Du côte de la théorie et de l'histoire}

A notre époque, la spécificité de la littérature de jeunesse, genre encore en émergence, est de plus en plus reconnue par les spécialistes de la théorie et l'histoire littéraires, soit qu'ils la regardent comme une littérature intentionnelle, écrite pour les enfants et portant en elle les indices de cette intentionnalité, soit qu'ils la considèrent comme ayant une double adresse, un double destinataire - enfants et adultes - et une épaisseur de significations, qui se dévoilent plus ou moins selon le degré de «pénétration » du lecteur, comme le dit si bien Charles Perrault.

Les hésitations entre les «étiquettes » à choisir pour nommer cette littérature spécifique montrent d'ailleurs sa complexité ; ainsi, par exemple, Johanna Prud'homme (2006) parle d'une littérature proprement intentionnlle, Roberta Pederzoli (2012) d'une littérature à double destinataire, tandis qu'Isabelle Nières-Chevrel (2012) l'appelle un « champ littéraire », Francis Marcoin, une "grande librairie » (2007) et Jean Perrot une « culture » pour enfants » (2011).

Les théoriciens et les praticiens de la traduction sont de plus en plus nombreux à reconnaître la spécificité de la traduction pour les enfants et à réfléchir à ce sujet. Parmi ceux qui ont publié des ouvrages et des articles sur cette riche problématique sont Riitta Oittinen, J.L.Coordonnier, Jean-Marc Gouanvic, Irina Mavrodin, Roberta Pederzoli, Chiara Elefante, Virginie Douglas, Mathilde Lévêque du côté des traductologues et François Matthieu, Denise Escarpit, Rose-Marie Vassalo, Marie-Claire Pasquier, Christiane Pintado, Claire Le Brun, Bertrand Ferrier, Fabio Regattin, Alina Pelea, Raluca-Nicoleta Balanchi, Daniela Hăisan et bien d'autres du côté des praticiens-théoriciens. La spécificité de ce type de traduction a été d'ailleurs signalée et reconnue par des traductologues de grande envergure comme Georges Mounin, Katharina Reiss ou Antoine Berman qui, de manière plutôt fugitive, ont affirmé que la traduction pour les enfants doit être considérée comme une catégorie à part, à côté de la traduction du théâtre, des textes religieux, des textes poétiques qui ont chacune leur créneau dans la littérature dite " générale». 
Une analyse et une critique encore timides des traductions de jeunesse se font distinguer ces dernières décennies et commencent à s'imposer dans des revues littéraires et surtout traductologiques. Nous pensons surtout nous au numéro spécial de la revue Méta, sous la direction de Riitta Oittinen, à plusieurs numéros de Translittérature, où la voix et les idées de François Matthieu et Rose-Marie Vassalo se font bien entendre, à deux numéros de Nous voulons lire, dirigés par Denise Escarpit, à une édition des Assises de traduction d'Arles ainsi qu'au dossier thématique d'un numéro de la revue Atelier de traduction, sous le patronage d'Irina Mavrodin, sans ignorer pour autant la contribution dans ce domaine d'autres revues et publications qui ont accordé une bonne ou petite place à la réflexion sur la traduction de la littérature de jeunesse.

Un bref regard dans l'histoire de la traduction (Constantinescu, 2012, 45-58) de ce genre vers le français est assuré par Isabelle Nières-Chevrel $(2012,665)$ pour qui, comme on l'a vu la littérature de jeunesse n'est pas un genre littéraire mais un " champs éditorial qui fédère des formes et des genres autour d'un lectorat ». Cette littérature destinée à un lecteur jeune ou réorientée vers lui connaît un développement "spectaculaire » au siècle dès le $\mathrm{XIX}^{\mathrm{e}}$ siècle et constitue un "secteur dynamique et rentable » avec une production largement diffusée (idem,666). Pour la première moitié du $\mathrm{XIX}^{\mathrm{e}}$ siècle la plupart des traductions viennent de l'Angleterre et de l'Allemagne et, comme le phénomène est important, des éditeurs-libraires commencent à se spécialiser dans ce domaine. Plusieurs aspects attirent l'attention des chercheurs comme le «triomphe » du chanoine bavarois Schmidt qui connaît un grand succès à son époque pour disparaître ensuite de la mémoire des lecteurs, les contes populaires et littéraires, dont ceux de Grimm et d'Andersen, et leur place importante dans les titres traduits pour le public jeune, le roman qui commence à gagner du terrain, en partant parfois des adaptations d'œuvres pour adultes, où l'on retient un titre comme la Case de l'oncle Tom de Harriet Beecher Stowe, qui seulement en deux ans jouit de dix traductions différentes. 
Un autre fait, qui nous intéresse particulièrement ici, est fréquent, courant même, notamment la " faible part de l'étranger " dans les traductions où les repères culturels de l'original sont, systématiquement, remplacés par des équivalents français dans la première moitié du siècle, tandis que dans la deuxième on signale déjà une ouverture à l'altérité. La présence/absence de l'étranger donne lieu à une intéressante analyse du parcours des patronymes de l'original vers le texte traduit où plusieurs solutions sont pratiquées : transposition graphique, traduction étymologique, francisation et même préservation. Le nom de la traductrice du Robinsson suisse - Elise Voïart - est à retenir pour le " discret marquage alémanique » de sa version et pour sa préface adressée aux jeunes lecteurs où elle leur explique son attitude envers les « étrangetés » et la « rudesse » de certains noms (idem, 686).

Très intéressante est la brève analyse qu' Isabelle Nières-Chevrel fait des procédés par lesquels les traducteurs allongent, expurgent ou procèdent à une sophistication du texte au nom d'un canon littéraire et des attendus de la culture d'arrivée.

Comme la traduction du conte domine au $\mathrm{XIX}^{\mathrm{e}}$ siècle, on peut parler d'une véritable " Europe des contes ", phénomène qui nous interesse particulièrement parce que le corpus choisi par nous concerne des contes, pris de plusieurs pays et continents de façon à pouvoir parler d'une véritable "planète des contes ».

Nous retenons aussi de ce bref regard historique qu'à la frontière entre le $\mathrm{XIX}^{\mathrm{e}}$ et le $\mathrm{XX}^{\mathrm{e}}$ sicèle s'impose aussi l'idée que traduire pour les enfants est un projet traductionnel spécifique, différencié du traduire pour les adultes qui pendule entre deux pôles : d'une part, l'attention du traducteur aux effets d'écriture de l'œuvre originale, étayée par la conviction que les livres de jeunesse relèvent pleinement de la littérature, d'autre part, le droit du traducteur d'évaluer ce qui convient ou non de donner à lire aux lecteurs et sous quelles formes, tendances encore vivantes tout au long du XIX ${ }^{\mathrm{e}}$ siècle et même, par endroits, dans la première moitié du $\mathrm{XX}^{\mathrm{e}}$ siècle.

Un regard historique sur la traduction pour les enfants est également donné par le Dictionnaire du livre de jeunesse ((Jean Perrot 
et Isabelle-Nières Chevrel, 2013) où les auteurs soulignent avec lucidité et honnêteté l'importance de la traduction dans la formation et le façonnage de la littérature qui accueille les traductions. On y souligne le rôle de la traduction dans l'histoire du livre de jeunesse comme "pourvoyeuse de modèles littéraires », " vecteur essentiel dans l'invention d'une littérature de fiction imaginée et écrite pour les enfants $»($ idem, 935).

De nos jours, à plusieurs reprises, des traducteurs pour enfants ont débattu ce complexe problème, ont exprimé leur credos et ont partagé leurs expériences uniques, en apparence, mais qui s'appuient sur des principes communs et des stratégies semblables, même si ajustées et adaptées d'un texte à l'autre, d'un auteur à l'autre, d'un espace culturel à l'autre. Une idée incontournable revient dans ces débats et confessions, bien synthétisée par Marc Parayre qui dit que traduire pour la jeunesse est « un vrai travail de traduction pour une littérature à part entière » (Parayre, 2007, 119)

Dans ce paysage varié de la réflexion sur la traduction pour le jeune public, où les voix des traductologues rencontrent celles des traducteurs, il est plutôt rare qu'un spécialiste en littérature de jeunesse écrive de façon très pertinente sur la manière dont elle est traduite vers d'autres langues. C'est le cas de Jean Perrot (2011), redoutable spécialiste en littérature de jeunesse, déjà évoqué, qui dans son livre, Du jeu des enfants et des livres à l'heure de la mondialisation, fait une très intéressante analyse des réflexions sur la traduction de la littérature de jeunesse et du contexte où ce phénomène se produit.

Jean Perrot identifie, avec finesse, le contexte nouveau au début du troisième millénaire où se déploie le processus de traduire pour les enfants, la polyphonie des langues nommées «singulières », en fait, moins connues et qui constitue un indice de la mondialisation, considérée sous son aspect positif, qui remplace le dialogue des langues dominantes, entre elles - anglais, français, allemand, espagnol -. Déjà au XVIII ${ }^{\mathrm{e}}$ et au XIX ${ }^{\mathrm{e}}$ siècles, la traduction des livres pour les enfants dans quelques langues de l'Europe a été, par les échanges réalisés, un moteur dans la construction du champ de la littérature d'enfance et de jeunesse. 
A présent les anciennes " hégémonies culturelles » ont été renversées et le japonais, l'arabe, le chinois, gagnent leur place dans la « citadelle linguistique », où pénètrent, petit à petit, également les langues régionales. Dans ces circonstances favorables c'est naturel d'avoir une version du Petit Prince en créole, une autre en amazighe, langue des berbères marocains. Grâce aux certaines initiatives des éditions comme Harmattan ou Unesco qui font paraître des livres bilingues, des langues réservées autrefois exclusivement à l'oralité, pénètrent dans la polyphonie des langues maternelles pour y inscrire leur différence et identité (Perrot, 2011, 71).

En prenant en compte cette nouvelle citadelle linguistique de la planète bien polyphonique où une mosaïque de langues et de cultures entrent, de plus en plus souvent, en dialogue, nous nous sommes arrêtée aux stratégies de traduction mises au service de la dimension culturelle du texte, point de départ pour de vrais échanges. Nous procédons dans la deuxième partie de notre article à quelques esquisses d'analyse, inspirées de la pratique en nous ralliant sans réserves à l'idée que « en principe, il n'est pas de traductologie qui vaille qui ne s'autorise expressément de son rapport à la prtique traduisante ». (Ladmiral, 2013, X)

\section{Du côté des pratiques}

L'analyse de plusieurs versions roumaines pour enfants des contes dits de France (Poveロti nemuritoare [Contes immortels], 1988), mais acclimatés et roumanisés à l'époque communiste de la censure et d'une idéologie envahissante, nous a alertée, il y a des années, sur un important éloignement de l'original. En suivant le principe simple de ne pas juger le travail du traducteur avant d'essaayer de le comprendre, "de retrouver le climat intellectuel qui fut le sien, de reconstituer son horizon de travail » (Chevrel, Masson, 2012, 12), nous accordons des circonstances atténuantes à cet éloignement de l'original. Il était pratiqué par les traducteurs, sous la pression idéologique de l'époque mais également au nom du pu- 
blic enfantin destinataire, et conduisait souvent à un de brouillage culturel du texte. Ce brouillage inacceptable au XXI ${ }^{\mathrm{e}}$ siècle, lorsque la mondialisation fait augmenter l'interêt pour l'identité culturelle, se produit lorsque le traducteur garde certains référents culturels mais procède à l'acclimatation d'autres, en créant la confusion et la déroute chez le petit lecteur.

Nous nous sommes rendu compte qu'il s'agit aussi d'un préjugé assez tenace et « international » concernant la compétence de lecture du jeune lecteur. C'est l'idée préconçue et très répandue que la traduction pour enfants, suppose nécessairement une simplification de l'écriture, une édulcoration du ton, une « mignardisation » de l'univers, comme si l'on voulait une « infantilisation » excessive du lecteur étranger qu'on suppose plus naïf, plus ignorant, plus sensible, plus délicat que le lecteur du texte original (Constantinescu, 2013b, 13-25). De plus, par la suppression des référents culturels du texte traduit, on maintient l'enfant dans un cocon linguistique et culturel qui le «protège » de toute étrangeté et étrangéité, de tout insolite, jugés sans doute, perturbateurs et compliqués pour le petit lecteur. Cela nous a incitée à une réflexion sur cette traduction à part et à une pratique des stratégies adéquates pour assurer l'ouverture de l'enfant à d'autres cultures et horizons. Et nous évoquons encore une fois les paroles de Ben Jelloun à propos du petit lecteur : "J'ai aimé me confronter aux enfants, écouter leurs questions, discuter avec eux. Cela vaut la peine, parce qu'un enfant est encore disponible pour accueillir des idées nouvelles. Les adultes ont des certitudes. Les enfants aiment apprendre. C'est un capital magnifique $[\ldots] »(2007,18)$

Ce « capital magnifique » doit être nourri, stimulé, entretenu pour qu'il puisse s'épanouir, entre autres à travers la lecture des contes étrangers qui font plonger le jeune lecteur dans des univers nouveaux, imprégnés de cultures différentes de la sienne. Le problème de la préservation de la dimension culturelle de l'original se pose donc lors de la traduction des contes pour les enfants surtout lorsque le public visé est d'un âge scolaire, de dix à douze ans. En prenant en compte les différences et les nuances exigées par les 
tranches d'âges, l'exercice de traduire des contes pour les enfants peut s'avérer une tâche plus difficile encore que traduire pour les adultes. Cela d'autant plus s'il s'agit des contes venant des peuples et cultures éloignés et peu connus dans l'espace culturel cible. Comme nous allons le voir, traduire pour les enfants est un processus complexe, très nuancé, un acte, à la fois, littéraire, pédagogique, didactique, culturel, éthique (Constantinescu, 2013a, 181)

Les contes choisis comme corpus pour les lectures traductologiques que nous proposons ici nous attirent et nous intéressent tout d'abord pour leur regard attentif accordé à la dimension culturelle du monde, sans ignorer pour autant celle symbolique, philosophique, étiologique, ludique etc. Ils paraissent chez des maisons d'éditions différentes : La tortue et l'araignée chez Isatis de Montréal, L'enfant perdu chez Nathan de Paris, Kulaguk et les géants chez Planète rebelle de Québec, L'orpheline, aux éditions Karthala et de l'Unesco, Paris, La petite fille et le pigeon chez Mulatinii de Suceava, Rêve noir d'un lapin blanc, chez Vents d'ailleurs, Paris, Le secret $d u$ potier chez Harmattan de Paris ${ }^{2}$ Ils ont en commun la relation entre identité et altérité, le respect de l'autre, respect qui se fait voir par l'emploi des termes renvoyant à des traditions et un patrimoine spécifiques créoles, amérindiens, inuit, bourkinabés, roumains, brésiliens, iraniens etc.

Ils ont été pour nous objet d'un travail déjà accompli, ou des projets traductifs à réaliser dans l'avenir. Ils appellent des stratégies variées comme le report des noms propres et des termes spécifiques (pour garder de la sorte les termes authentiques), l'incrémentialisation par périphrase explicitante (pour éclairer le jeune lecteur et l'aider dans sa compréhension), la translittération et l'adaptation phonétique des anthroponymes et toponymes (pour rendre lisibles des noms à orthographe difficile pour un jeune lecteur étranger) (Ballard 2003, 154-157). A cela s'ajoute un brin de créativité et un appareil paratextuel, conçu de façon didactique, qui offre des renseignements complémentaires, adaptés à l'âge jeune, car comme le dit Ben Jelloun « les enfants aiment apprendre ». Ce n'est donc pas une attitude appropriée de les tenir « à l'écart des rapports d'alté- 
rité », comme s'ils étaient « des êtres particulièrement fragiles, qui ne peuvent pas comprendre. » (Coordonnier, 1995, 15).

Nous commençons la partie analytique par le conte créole La tortue et l'araignée, raconté par Dinah Psyché, paru chez Isatis. C'est l'histoire d'une tortue débrouillarde et maligne qui sait bien profiter des autres pour obtenir ce qu'elle veut, en allant même jusqu'à l'imprudence. Cette image de la tortue contredit celle répandue dans l'espace européen, qui obéit au principe latin de "festina lente », et d'une sage bête qui vainc le lièvre parce qu'elle "se hâte avec lenteur » et observe un principe de bon sens : « rien ne sert de courir, il faut partir à temps », comme nous l'a raconté La Fontaine. Le conteur parsème son conte sur Toti la rusée qui profite trop de Zagryien l'araignée, par des formulettes de contage qui maintiennent éveillée l'attention du lecteur/ auditeur (à l'origine) comme Kouté pou konprann (écoutez pour comprendre), structure phatique qui doit être gardée, par report, dans le texte cible pour ce qu'on pourrait appeler sa " musicalité identitaire ", tout comme les refrains listikrik, listikrak ou krik, krak, yékrik, yékrak, phatiques et ludiques, à la fois Ces derniers contribuent au rythme alerte du conte et de son contage et satisfont, sans doute, l'attente ludique des jeunes lecteurs.

En dehors des formulettes, traces indubitables de l'oralité originaire du conte, Dinah Psyché émaille son histoire de termes créoles - ababa, bèt-a-plim, grigri, katjopin, zagriyen - dont le sens est éclairé par le contexte. Mais pour satisfaire la curiosité encyclopédique du jeune lecteur, ces termes sont également expliqués par un lexique bilingue créole-français qui lors de la traduction vers une autre langue cible pourrait être gardé ; les traductions-explications seraient parfois adaptées à une autre réalité géographique. Par exemple, des termes comme quimbois, signifiant sorcellerie pratiquée en Martinique, peut être gardé tel quel et son explication traduite sans ajustements, de même que $z u k$, signifiant bal ; en échange, un terme comme $k w i$ peut être explicité et ajusté par une structure moins étendue que dans l'original, du type « sorte de demi calebasse avec la peau très lisse » pour souligner justement le trait spécifique pour la carapace de la tortue avant sa chute. 
La collection Korrigan des éditions Isatis est conçue et structurée de façon à satisfaire la curiosité du lecteur jeune, désireux de « connaître davantage », comme s'intitulent les Annexes, mais sans l'obliger à interrompre sa lecture pour cela. Le contexte verbal et iconique éclaire suffisamment le contenu des termes et l'histoire peut être comprise et suivie également sans la lecture des annexes, qui comportent le lexique déjà mentionné, des notions sur les différences entre conte, mythe et légende, plusieurs explications sur le conte animalier ainsi que les brèves présentations de l'auteure Dinah Psyché et de l'illustratrice Annouchka Galouchko.

La même attention pour l'identité de l'autre, pour la curiosité du jeune lecteur masi aussi l'intérêt accordé au développement de sa compétence encyclopédique se retrouvent dans la collection «contes et légendes », surnommée "collection de la mémoire du monde » chez les éditions Nathan, où paraît le conte amérindien L'enfant per$d u$, raconté par Charles Le Blanc, traductologue mais aussi conteur, à ses heures perdues. Il est d'ailleurs présenté de façon attrayante pour les enfants comme quelqu'un qui, petit, n'aimait pas l'école, grand, s'est marié avec une belle princesse des Abruzzes et qui, à présent, écrit aidé par un magnifique chat blanc, Mélisande.

Le conte amérindien présente l'histoire d'un enfant d'Abénakis, égaré de ses parents et adopté par une famille d'ours. Comme ses parents biologiques meurent dans une coulée de boue, l'enfant élevé par des ours s'attache bien à ses « parents » adoptifs et apprend et pratique leur langage et leurs habitudes. Récupéré après un certain temps par les voisins au prix de la mort de ses parents-ours, l'enfant perdu, doué d'une force presque miraculeuse, reste avec les hommes, tout en gardant un lien fort avec les animaux, en étant une sorte de médiateur entre ces derniers et les premiers. La légende dit que cet être intermédiaire, au propre et au figuré, était, en fait, un esprit bénéfique que le «manitou » avait envoyé aux Abénakis. Le « petit abécédaire pour mieux lire ce conte » éclaire le lecteur sur le terme amérindien « manitou » qu'il avait, sans doute, un peu deviné et sur d'autres comme, «tomahawk », " orignal », « masquinongé » ou « ouananiche », gardés par report par le conteur au chat blanc. 
C'est, croyons-nous, une bonne façon d'apprivoiser une langue, de faire un premier contact avec son étrangeté et son étrangéité.

L'adaptation du conte inuit Kulaguk et les géants réalisée par Pascale Desbois, publié chez Planète rebelle, Québec, et accompagné d'un $\mathrm{CD}$, fait plonger son jeune lecteur dans l'univers particulier des chasseurs inuit de phoques, terrorisés par un géant dévoreur et par sa femme. Les deux êtres monstrueux vont être vaincus par la ruse et l'intelligence du chasseur Kulaguk. L'explosion de la géante, qui par sottise tombe dans le piège du chasseur et boit toute une rivière, conduit à la naissance du brouillard. L'histoire étiologique de l'exploit de Kulaguk fait comprendre aux enfants d'une langue et culture éloignées des termes comme "igloo », " toundra », " babiche », éclairés bien par le contexte, ayant le statut d'emprunt ou de report, selon la proximité et l'éloignement des deux langues, source et cible. A cela contribuent également les illustrations très suggestives de Frédérique Lafortune et la lecture ensorcelante de Stéphanie Vecchio qui les deux «traduisent » le conte à leur façon.

Le conte bourkinabé l'Orpheline, traitant du conflit entre la belle fille et la marâtre, traduit en français par Alain Sissao depuis la langue moore, est très stimulant pour le traducteur vers une autre langue sous le même aspect de la présence des vers, comptines, chansons, expressions en langue moore. Ils ont été gardés tels quels dans la version française et donnés en caractères italiques ou entre guillemets par le collecteur de contes et chercheur Alain Sissao (Constantinescu, 2007, 23-34). Ces apparents points d'opacité du texte, ces "verres colorés » par une culture étrange et étrangère pour le public d'accueil sont la meilleure façon de donner accès au lecteur à cette « étrangeté » qu'il ignorait auparavant. On les a préservés dans le nouveau texte cible car le contexte prenait très bien en charge le sens, par exemple, de la chanson : " L'orpheline se leva. En pleurant, elle se dirigea vers le champ de gombo en chantant : 'Sî lengen lengen leende !'. Il faut remarquer d'ailleurs que seulement le refrain de la première chanson est rendu en moore, étant intégré dans l'ensemble de la chanson qui est 
longue et a aussi un contenu narratif. En revanche, la deuxième chanson, très courte, est rendue intégralement en original et malgré le contexte éclairant ( La fille partit en pleurant et en chantant») est accompagnée d'une note qui précise : «Chant de lamentation », note, superflue, selon nous. Pour préserver la même couleur locale, évoquée aussi par le paysage de la brousse, on n'pas traduit le terme gombo - désignant une plante tropicale - parce que le contexte "semer du gombo », " le gombo arriva à maturité et il était prêt à récolter », " récolter le gombo », indiquait bien le terme générique, une plante. On a adopté la même solution pour le terme sorgho, autre plante tropicale. On n'a pas donné de notes explicatives en bas de page, justement pour ne pas rompre la tension épique et ne pas distraire l'attention du lecteur vis-à-vis du récit.

Pour développer et épanouir la compétence encyclopédique du jeune lecteur, on peut prévoir, lors de la publication en volume, un lexique explicatif en annexe et, au cas de la publication en une collection de jeunesse, des illustrations plus éclairantes encore que tout glossaire car elles donneraient une « image », au sens propre du mot, d'un paysage différent de celui du lecteur, d'un cadre inédit, qui peut sembler, dans un premier temps, inhabituel ou bizarre.

$\mathrm{Au}$ moins deux termes roumains, porteurs d'étrangeté et d'étrangéité seraient à comprendre et apprendre de l'histoire $L a$ Petite fille et le pigeon de Doina Cernica, traduite en français, qui raconte la bonne rencontre de la Petite Fille et du Pigeon qui se lient d'amitié et aident ensemble les deux enfants endeuillés par la mort de leur grand-mère. D'abord le terme coliva, éclairé bien par incrémentialisation, à travers une périphrase explicitante : "le gâteau des morts pour les vivants » et dont on apprend d'autres détails comme : «sucré et délicieux, pour évoquer les disparus avec bonté et douceur. On le cuisine avec du miel et du blé. » Ce terme est important parce qu'il renvoie à une pâtisserie spécifique, à valeur symbolique, pour des rituels traditionnels encore persistants en l'espace roumain.

L'autre terme à retenir est le nom dor qui dans l'expression « avoir dor» exprime « la nostalgie et le regret ressentis par l'ab- 
sence de quelqu'un ou de quelque chose ». Ce mot à forte charge symbolique est présent également dans la poésie populaire du pays et il est essentiel dans la compréhension d'une certaine spiritualité roumaine. Nous pensons que le terme portugais «saudade », avec un contenu sémantique semblable mais riche en connotations peu traduisibles aurait mérité d'être gardé dans une histoire comme la Petite fille et la mer de Sophia De Mello Breyner où la traductrice a préféré l'équivalent français « nostalgie », qui n'as pas une charge symbolique tout aussi importante.

Une suggestion de bonne solution vient, en échange, de la traduction du conte Rêve noir d'un lapin blanc, d'Ana Maria Machado où le mot portugais Menina est gardé mais en compagnie de son équivalent français, la fillette, donnant ainsi une touche de couleur culturelle spécifique à ce beau récit brésilien.

Une expression comme «le soufflement du potier » pourrait marquer le petit lecteur du conte Le secret $d u$ potier, bilingue français-persan, adapté et merveilleusement illustré par Claire Jobert. Si le persan n'est pas d'un accès facile pour un jeune lecteur européen, par exemple, des sonorités spécifiques à cette langue passent au moins dans les noms des personnages, adaptés phonétiquement : d'une part, Oustâ Kazêm, le maître apprenti et, d'autre part, Noureddine, son apprenti et l'héritier de son savoir. Les toponymes Qom et Hamadan, représentant les lieux importants pour la fabrication des poteries, s'inscrivent eux aussi parmi les termes à charge culturelle. Le secret que le maître apprend à son élève - il faut, à la fin du travail, un soufflement pour enlever la poussière qui ternit les couleurs - est une belle leçon sur l'importance de connaître « les ficelles du métier » et, métaphoriquement sur le rôle $\mathrm{du}$ « soufflement » dans tout travail fait avec passion.

Qu'il s'agisse de termes spécifiques, de noms à sonorités particulières, de refrains ludiques, de formulettes musicales ou d'expressions sapientiales, ces unités sont de véritables «pépites » des contes choisis comme corpus qui méritent d'être précieusement gardées à travers diverses stratégies de traduction, adaptées au contexte, à l'âge, à la créativité du traducteur etc. 


\section{Conclusion}

Les chercheurs sont d'accord et le répète souvent : "On traduit toujours en contexte » (Froeliger, 2013, 11). C'est ce que nous avons essayé de démontrer dans ce qui précède et ce qui explique une massive adaptation et accimatation du texte pour enfants aux siècles passés et une nouvelle vision traductive à partir de la deuxième moitié du $\mathrm{XX}^{\mathrm{e}}$ siècle. Les mentalités changes, les préjugés sur la fragilité du lecteur enfants ne sont plus de mise de nos jours. A l'époque où, de plus en plus, la dimension culturelle et civilisationnelle d'une communauté est valorisante et on cherche à lui donner la reconnaissance qu'elle mérite, la traduction pour les enfants, "futurs citoyens du monde » et non pas " de simples consommateurs » (Perrot, 2011, 426), ne peut pas ignorer ce phénomène où l'identité s'épanouit par et dans son rapport à l'altérité. Ces quelques réflexions sur la manière de préserver la dimension culturelle d'un conte adressé à un jeune public, filtrées par les pratiques et les projets du traducteur, se veulent des suggestions de solutions dans le travail traductif. Ce sont pour ainsi dire de models présumés utiles, de possibles exemples à suivre dans le travail de longue haleine d'ouvrir les enfants à des cultures nouvelles, à travers la traduction, de les aider à développer leur « capital magnifique » de lecteurs qui « aiment apprendre ».

\section{Notes}

1. Article élaboré dans le cadre du projet PN-II-ID-PCE-2011-3-0812, Traduction culturelle et littérature(s) francophone(s): histoire, réception et critiques des traductions. 
2. Nous reprenons dans ce qui suit certaines idées développées dans notre ouvrage Lire et traduire la littérature de jeunesse - des contes de Perrault aux textes ludiques contemporains, P.I.E. Peter Lang, Bruxelles, 2013b, 179-182. Elles concernent des suggestions d'analyse pour les contes La tortue et l'araignée, L'enfant perdu, Kulaguk et les géants, La petite fille et le pigeon chez Mulatinii de Suceava, Le secret du potier chez Harmattan de Paris

\section{Sources:}

Cernica, Doina. Fetița și porumbelul/La petite fille et le pigeon. Trad. Muguraș, Constantinescu, Suceava : Editura Mușatinii, 2011.

De Mello Breyner, Sophia, La Petite Fille de la Mer, traduit du portugais par Natalia Vital, Paris : Éditions de la Différence, 1999.

Desbois, Pascale. Kulaguk et les géants in Contes traditionnels du Canada, (adaptés par), accompagné de CD, Québec : Planète rebelle, 2003.

Jobert, Claire, Le secret du potier.Bbilingue français-persan (adapté d'un conte traditionnel et illustré par), Paris : L'Harmattan.

Le Blanc, Charles. L'enfant perdu (un conte amérindien par). Contes et légendes de la nature enchantée, Paris : Nathan, 2003.

Machado, Ana-Maria . Rêve noir d'un lapin blanc, trad. Dominique Boisdron, Sylvie Gradel, Vents d'ailleurs, 2005.

Povești franceze. Povești nemuritoare, trad. Dana Oprita, $n^{\circ} 220$, București : Editura Ion Creangă, 1988.

Psyché, Dinah. La tortue et l'araignée, conte créole. Montréal : Éditions de l'Isatis, coll. Korrigan, 2005.

Sissao, Alain. Contes du pays des Moose - Bourkina Faso. Paris : Éditions KARTHALA - Éditions UNESCO, 2002. 


\section{Références}

*** Atelier de traduction, Dossier : La traduction de la littérature de jeunesse, $\mathrm{nr}$. 8, 2007, Editura Universității Suceava, Suceava, 2007.

Balațchi, Raluca-Nicoleta. «Sans famille en roumain : retraductions et rééditions », Douglas, Virginie; Cabaret, Florence (dir./eds.), La retraduction en littérature de jeunesse/ Retranslating Children's Literature. Collection Recherches comparatives sur les livres et le multimedia d'enfance, $\mathrm{n}^{\circ}$ 7, P.I.E. Peter Lang, Bruxelles, 2014, ISBN 978-90-5201-710-5, 151-164.

Ballard, Michel. Versus : la version réfléchie. Repérages et paramètres. Ophrys, 2003.

Ballard, Michel. Le nom propre en traduction, Gap : Ophrys, 2001.

Ben, Jelloun ; Tahar, Entretien (avec E.B. Steiciuc). Atelier de traduction, Dossier : La traduction de la littérature de jeunesse, n. 8, 2007, Editura Universității Suceava, Suceava, 2007, 15-22.

Berman, Antoine. Pour une critique des traductions : John Donne. Paris : Gallimard, 1995.

Berman, Antoine. L'épreuve de l'étranger, Paris : Gallimard, 2002.

Cabaret, Florence ; Virginie, Douglas. (sous la diection), La Retraduction en littérature de jeunesse / Retranslating Children's Literature. Bruxelles: P.I.E. Peter Lang, 2014, ISBN 978-90-5201-710-5.

Chevrel, Yves ; Masson, Jean Yves. «Avant-propos ». Chevrel, Yves ; D’hulst, Lieven ; Lombez, Christine . Histoire des traductions en langue française - Dixneuvième siècle (1815-1914). Paris : Verdier, 2012, 7-14.

Constantinescu, Muguraș, "Traduire pour les enfants des contes moose et inuit ». Atelier de traduction, Dossier : La traduction de la littérature de jeunesse, nr. 8, 2007, Suceava : Editura Universității Suceava, 2007, 23-34. 
Constantinescu, Muguraș. "Une renversante histoire des traductions en langue française ». Atelier de traduction, n. 18, 2012, Editura Universităţii din Suceava, ISSN 2344-5610, ISSN-L 1584-1804, 45-58.

Constantinescu, Muguraș. " La traduction de la dimension culturelle dans la littérature de jeunesse ". Littérature pour la jeunesse et diversité culturelle, Virginie Douglas (sous la direction de), Paris : Harmattan, 2013a (173-187).

Constantinescu, Muguraş. Lire et traduire la littérature de jeunesse - des contes de Perrault aux textes ludiques contemporains. Bruxelles : P.I.E. Peter Lang, $2013 b$.

Coordonnier, Jean Louis. Traduction et culture. Hatier Didier : LAL, 1995.

Cabaret, Florence ; Virginie, Douglas (sous la diection). La Retraduction en littérature de jeunesse / Retranslating Children's Literature. Bruxelles : P.I.E. Peter Lang, 2014, ISBN 978-90-5201-710-5.

Di Giovanni, Elena ; Elefante, Chiara ; Pederzoli, Roberta. Écrire et traduire pour les enfants/Writing and Translating for Children . Bruxelles: Peter Lang, 2010.

Douglas, Virginie (sous la direction). Littérature pour la jeunesse et diversité culturelle, Paris : Harmattan, 2013.

Froeliger, Nicolas. Les noces de l'analogique et du numérique - De la traduction pragmatique. Paris : Les Belles Lettres, coll. Traductologiques, 2013.

Hăisan, Daniela. „La retraduction: miroir magique, boîte catoptrique ou kaléidoscope. Poil de Carotte et les sept versions roumaines", in Douglas, Virginie; Cabaret, Florence (dir./eds.), La retraduction en littérature de jeunesse / Retranslating Children's Literature, Recherches comparatives sur les livres et le multimedia d'enfance $\mathrm{n}^{\circ}$ 7, P.I.E. Peter Lang, Bruxelles, 2014, ISBN 978-905201-710-5, 169-182.

Ladmiral, Jean-René. "Préface ", in Les noces de l'analogique et du numérique De la traduction pragmatique. Paris : Les Belles Lettres, coll. Traductologiques, 2013, VII-XIV. 
Lévêque, Mathilde. Écrire pour la jeunesse en France et en Allemagne dans l'entre-deux-guerres. Rennes : Presses universitaires de Rennes, 2011.

Marcoin, Francis (en collaboration avec Chelebourg, Christian). La littérature de jeunesse. Paris : Armand Colin, 2007.

Mavrodin, Irina. Despre traducere. Literal i în toate sensurile. Craiova : Scrisul Românesc, 2006.

Mounin, Georges. Les belles infidèles. Lille : Presses Universitaires de Lille, 1994.

Nières-Chevrel, Isabelle. "Littérature d'nfance et de jeunesse ». Chevrel, Yves ; D'hulst, Lieven ; Lombez, Christine. Histoire des traductions en langue française - Dix-neuvième siècle (1815-1914). Verdier, Paris, 2012, 665-726.

Nières-Chevrel ; Isabelle, Perrot (sous la direction). Dictionnaire du livre de jeunesse. Paris : Editions du Cercle de la Librairie, 2013.

Pederzoli, Roberta. La traduction de la littérature d'enfance et de jeunesse et le dilemme du destinataire. Bruxelles : P.I.E. Peter Lang, 2012.

Prud'homme, Johanna. "La traduction en littérature pour la jeunesse : une interface à double tranchant ». Rabat : CCLMC, 2006, 165-172.

Méta, Traduction pour les enfants / Translation for children (sous la direction de Riitta Oittinen), Volume 48, numéro 1-2, Mai 2003, p. 1-327, Les Presses Universitaires de Montréal, Montréal, 2003.

Nous voulons lire, n.171, 2007, NVL/CRALE, Bordeaux.

Nous voulons lire, n.172, 2007, NVL/CRALE, Bordeaux.

Palimpsestes, n.11, Traduire la culture, Presses de la Sorbonne Nouvelle, Paris, 1998. 
Translittérature n. 13, été 97, Dossier « Traduire pour la jeunesse », Paris, 1997.

Michaud, Henriette. « Du conte oral au conte écrit». Translittérature no. 18 -19, printemps 2000, Dossier « Il était une fois : traduire le conte », Paris, 2000.

Parayre, Marc. "Un vrai travail de traduction pour une littérature à part entière », in Atelier de traduction nr. 8, Editura Universităţii Suceava, Suceava, 2007, 119-128.

Perrot, Jean. Du jeu, des enfants et des livres à l'heure de la mondialisation. Bruxelles : Éditions du Cercle de la Librairie, Paris, 2011.

Reiss, Katharina. La critique des traductions, ses possibilités et ses limites. traduit de l'allemand par C. Bocquet, Artois Presses Université, 2002.

Recebido em: 05/08/2015 Aceito em: 15/10/2015 